\title{
EVOLUTION OF THE NUCLEAR ACCRETION DISK EMISSION IN NGC 1097: GETTING CLOSER TO THE BLACK HOLE
}

\author{
Thaisa Storchi-Bergmann ${ }^{1,2}$ and Rodrigo Nemmen da Silva \\ Instituto de Física, Universidad Federal do Rio Grande do Sul, Campus do Vale, Porto Alegre, RS, Brazil; \\ thaisa@if.ufrgs.br \\ Michael ERaCleous $1,3,4$ \\ Department of Astronomy and Astrophysics, Pennsylvania State University, \\ 525 Davey Lab, University Park, PA 16802 \\ Jules P. HALPERN ${ }^{1}$ \\ Columbia Astrophysics Laboratory, Columbia University, 550 West 120th Street, New York, NY 10027 \\ ANDREW S. WILSON ${ }^{1}$ \\ Astronomy Department, University of Maryland, College Park, MD 20742 \\ Alexei V. FilipPenKo 5,6 \\ Department of Astronomy, University of California, Berkeley, CA 94720-3411 \\ Maria Teresa Ruiz ${ }^{1}$ \\ Departamento de Astronomía, Universidad de Chile, Casilla 36-D, Santiago, Chile \\ R. CHRIS SMITH \\ Cerro Tololo Inter-American Observatory, National Optical Astronomy Observatory, Casilla 603, La Serena, Chile \\ AND \\ NEIL M. NAGAR ${ }^{1}$ \\ Arcetri Observatory, Largo E. Fermi 5, Florence 50125, Italy \\ Received 2003 May 8; accepted 2003 August 3
}

\begin{abstract}
We study the evolution of the broad, double-peaked $\mathrm{H} \alpha$ emission-line profile of the LINER/Seyfert 1 nucleus of NGC 1097, using 24 spectra obtained over a time span of 11 years-from 1991 November through 2002 October. While in the first 5 years the main variation was in the relative intensity of the blue and red peaks, in the last years we have also observed an increasing separation between the two peaks, at the same time as the integrated flux in the broad line has decreased. We propose a scenario in which the emission originates in an asymmetric accretion disk around a supermassive black hole, whose source of ionization is getting dimmer, causing the region of maximum emission to come closer to the center (and thus to regions of higher projected velocity). We use the observations to constrain the evolution of the accretion disk emission and to evaluate two models: the elliptical-disk model previously found to reproduce the observations from 1991 to 1996 and a model of a circular disk with a single spiral arm. In both models the peak emissivity of the disk drifts inward with time, while the azimuthal orientation of the elliptical-disk or the spiral pattern varies with time. In the case of the spiral-arm model, the whole set of data is consistent with a monotonic precession of the spiral pattern, which has completed almost two revolutions since 1991. Thus, we favor the spiral-arm model, which, through the precession period, implies a black hole mass that is consistent with the observed stellar velocity dispersion. In contrast, the elliptical-disk model requires a mass that is an order of magnitude lower. Finally, we have found tentative evidence of the emergence of an accretion disk wind, which we hope to explore further with future observations.
\end{abstract}

Subject headings: accretion, accretion disks — galaxies: individual (NGC 1097) — galaxies: nuclei — galaxies: Seyfert — line: profiles

\section{INTRODUCTION}

The barred spiral galaxy NGC 1097 was the first example of a LINER (low-ionization nuclear emission-line region; Heckman 1980) to display broad, double-peaked Balmer lines. The double-peaked $\mathrm{H} \alpha$ line $\left(\mathrm{FWHM} \approx 7500 \mathrm{~km} \mathrm{~s}^{-1}\right)$ was discovered by Storchi-Bergmann, Baldwin, \& Wilson (1993, hereafter SB93) after a careful subtraction of the starlight from the nuclear spectrum. The broad line was not easily recognizable in the original spectrum, because its

\footnotetext{
${ }^{1}$ Visiting Astronomer, Cerro Tololo Inter-American Observatory, which is operated by the Association of Universities for Research in Astronomy (AURA), Inc., under contract to the National Science Foundation.

${ }^{2}$ Visiting Astronomer, European Southern Observatory, La Silla, Chile.

${ }^{3}$ Visiting Astronomer, Kitt Peak National Observatory, which is operated by AURA, Inc., under contract to the National Science Foundation.

${ }^{4}$ Visiting Astronomer, MDM Observatory.

${ }^{5}$ Visiting Astronomer, W. M. Keck Observatory, operated by the California Institute of Technology, the University of California, and NASA.

${ }^{6}$ Visiting Astronomer, Lick Observatory.
} 
contrast against the continuum from the underlying stellar population was fairly low. This discovery was followed by other examples, all of them in Hubble Space Telescope (HST) spectra taken through narrow apertures in which starlight from the host galaxy is largely excluded. These examples include M81 (Bower et al. 1996), NGC 4203 (Shields et al. 2000), NGC 4450 (Ho et al. 2000), and NGC 4579 (Barth et al. 2001).

Comparison of the double-peaked $\mathrm{H} \alpha$ spectrum of NGC 1097 with earlier observations, as well as the later evolution of the double-peaked profile, led to the conclusion that the broad Balmer lines were associated with a transient event (see, e.g., SB93; Eracleous et al. 1995; StorchiBergmann et al. 1995, 1997; hereafter SB95, SB97), presumably an episode of mass accretion onto the nuclear supermassive black hole (SMBH).

The recent findings of a proportionality between the mass of galactic bulges and that of the SMBHs in galaxies that are close enough to allow the SMBHs' detection via stellar kinematics (see, e.g., Ferrarese \& Merritt 2000; Gebhardt et al. 2000) support their presence in the nuclei of most present-day galaxies. Such an SMBH will eventually accrete mass, for example, via the capture of a star passing closer to the black hole than its tidal radius. If the SMBH has a mass smaller than $\sim 10^{8} M_{\odot}$, a solar-mass star will be tidally disrupted before it is accreted, leading to the formation of an accretion disk or ring. As argued by SB95 and SB97, such a process is the preferred interpretation for the double-peaked lines of NGC 1097 and may also be applicable to some of the other LINERs mentioned above. It is thus possible that we have begun to witness such tidal disruption events, which have long been predicted by theory (see review by Rees 1988).

Monitoring the variability of the double-peaked profiles provides useful constraints on the above scenario. Therefore, we have been following the evolution of the $\mathrm{H} \alpha$ profile of NGC 1097 since it was first discovered in 1991. In this paper we present the result of 11 years of monitoring and use observations of the profiles to constrain and refine scenarios for their origin, such as the precessing elliptical ring suggested by Eracleous et al. (1995) and SB95.

In $\S 2$ we describe the observations and analysis of the data, in $\S 3$ we present the results, and in $\S 4$ we discuss their interpretation and present two possible accretion disk models to reproduce the profile variations. We explore in $\S 5$ the significance of our findings and compare them with those for other objects. The conclusions of this work are presented in $\S 6$.

\section{OBSERVATIONS}

We observed the nuclear spectrum of NGC 1097 from 1991 to 2002, up to several times a year, using mostly the Cerro Tololo Inter-American Observatory (CTIO) $4 \mathrm{~m}$ Blanco and $1.5 \mathrm{~m}$ telescopes and the KPNO $2.1 \mathrm{~m}$ telescope. We also obtained a few spectra with the Keck II, Lick 3 m, and MDM $2.4 \mathrm{~m}$ telescopes and the ESO New Technology Telescope (NTT). The log of observations is given in Table 1, where we also list the slit and extraction window widths.

The first observations (SB93, SB95, SB97) were obtained through a $2^{\prime \prime}$ slit and an extraction window of $4^{\prime \prime}$. We later used a narrower slit of 1 .'5 for the observations with the large telescopes (3.6 and $4 \mathrm{~m}$ ), in order to improve the
TABLE 1

OBSERVATION LOG

\begin{tabular}{|c|c|c|c|c|}
\hline Date & Telescope & $\begin{array}{l}\text { Exposure } \\
\text { (s) }\end{array}$ & $\begin{array}{c}\text { Aperture } \\
(\operatorname{arcsec})\end{array}$ & $\begin{array}{l}\text { Scaling } \\
\text { Factor }\end{array}$ \\
\hline 1991 Nov. $2 . . \ldots \ldots . .$. & CTIO $4 \mathrm{~m}$ & 2700 & $2.0 \times 4.0$ & 1.12 \\
\hline 1992 Oct. 5 .......... & CTIO 4 m & 685 & $2.0 \times 4.0$ & 1.32 \\
\hline 1993 Sep. 12 ........ & Lick 3 m & 1200 & $2.0 \times 4.0$ & 0.52 \\
\hline 1994 Jan. 5 .......... & CTIO 4 m & 1800 & $2.0 \times 4.0$ & 1.14 \\
\hline 1994 Dec. $6 \ldots \ldots \ldots .$. & CTIO 4 m & 1200 & $1.5 \times 3.7$ & 1.46 \\
\hline 1996 Jan. 24 ....... & CTIO 4 m & 1200 & $2.0 \times 4.0$ & 1.00 \\
\hline 1996 Oct. $10 \ldots \ldots$. & Lick 3 m & 3600 & $2.0 \times 4.7$ & 0.91 \\
\hline 1997 Jan. 2 .......... & CTIO $1.5 \mathrm{~m}$ & 3600 & $1.8 \times 2.6$ & 0.66 \\
\hline 1997 Sep. 24 ....... & CTIO 4 m & 1800 & $1.5 \times 3.7$ & 1.49 \\
\hline 1997 Sep. 27 ........ & KPNO $2.1 \mathrm{~m}$ & 3600 & $1.9 \times 3.8$ & 1.22 \\
\hline 1998 Jan. 2 .......... & CTIO 1.5 m & 6000 & $1.8 \times 2.6$ & 1.79 \\
\hline 1998 Jan. 17 ........ & Keck II & 500 & $1.0 \times 4.8$ & 1.28 \\
\hline 1998 Feb. 1.......... & CTIO 4 m & 1800 & $1.5 \times 3.7$ & 1.27 \\
\hline 1998 Oct. $15 \ldots \ldots$ & KPNO $2.1 \mathrm{~m}$ & 5400 & $1.9 \times 4.6$ & 1.42 \\
\hline 1998 Oct. $20 \ldots \ldots$. & CTIO 1.5 m & 5400 & $1.8 \times 6.5$ & 0.87 \\
\hline 1998 Dec. $16 \ldots \ldots .$. & MDM $2.4 \mathrm{~m}$ & 2400 & $1.5 \times 2.9$ & 1.19 \\
\hline 1998 Dec. $28 \ldots \ldots .$. & CTIO 4 m & 1800 & $1.5 \times 3.7$ & 1.29 \\
\hline 1999 Jan. 1 .......... & Keck II & 300 & $1.0 \times 2.4$ & 1.11 \\
\hline 1999 Dec. $2 \ldots \ldots \ldots$ & KPNO $2.1 \mathrm{~m}$ & 3600 & $1.9 \times 3.0$ & 1.21 \\
\hline 2000 Sep. $23 \ldots \ldots$. & KPNO $2.1 \mathrm{~m}$ & 3600 & $1.9 \times 3.0$ & 1.10 \\
\hline 2000 Oct. $11 \ldots \ldots$. & Keck II & 300 & $1.0 \times 2.4$ & 0.70 \\
\hline 2001 Mar. 1 ......... & CTIO 4 m & 900 & $1.5 \times 3.7$ & 1.22 \\
\hline 2001 Sep. 14 ........ & NTT 3.6 m & 1800 & $1.5 \times 3.7$ & 1.33 \\
\hline 2002 Oct. $10 \ldots \ldots$. & KPNO $2.1 \mathrm{~m}$ & 3600 & $1.9 \times 3.0$ & 1.17 \\
\hline
\end{tabular}

contrast between the double-peaked profile and the starlight spectrum, but kept it wide enough to avoid spurious variations due to seeing differences. We have also kept the extraction window large enough to allow normalization of the double-peaked broad-line flux using the fluxes of the narrow emission lines, as discussed below.

In order to isolate the double-peaked profile, we subtracted the contribution of the stellar population, as described in SB93, SB95, and SB97. In the case of the observations with the Blanco and NTT telescopes, the stellar population spectrum was extracted from windows adjacent to the nuclear window, typically from regions between $3^{\prime \prime}$ and $6^{\prime \prime}$ from the nucleus. The nuclear spectrum is redder than that of the stellar population at these windows. Attributing the difference in color to reddening by dust, we estimate a value of $E(B-V) \approx 0.05 \mathrm{mag}$. We have thus reddened the stellar population spectra accordingly and normalized them to the flux of the continuum in the nuclear spectrum. In addition, we have excised the residual narrow-line emission from the stellar population spectra (using as references spectra of disk galaxies free of emission lines) and have subtracted it from the nuclear spectrum. The spectra obtained with the smaller telescopes (CTIO $1.5 \mathrm{~m}$ and KPNO $2.1 \mathrm{~m}$ ) have signal-to-noise ratios too low outside the nucleus to allow the extraction of a population template; for these we used the starlight spectra extracted from the CTIO $4 \mathrm{~m}$ observations to subtract the stellar population contribution. The same was done for the Keck and MDM spectra. The process of starlight subtraction is more important in the most recent spectra because the doublepeaked line is fading (see discussion below), and its contrast to the stellar population spectrum is decreasing. Thus, a careful match of the stellar population spectrum is necessary before the subtraction. 
The last step was to normalize the broad-line flux, for which we used the fluxes of the narrow emission lines $\mathrm{H} \alpha$, [N II] $\lambda \lambda 6548,6584$, and [S II] $\lambda \lambda 6717,6731$, under the assumption that they have not varied during the 11 years of observations. This is a reasonable assumption, considering that the average extraction windows correspond to $\sim 150$ pc $\times 300$ pc at the galaxy. For the normalization we used as reference the spectrum of 1996 January 24, as follows: For each spectrum we determined the average ratio of the $[\mathrm{N} \mathrm{II}]+\mathrm{H} \alpha$ (narrow) and [S II] fluxes with respect to the 1996 January 24 spectrum and multiplied the spectrum by this average ratio. This scaling factor is listed for each spectrum in the last column of Table 1 . The success of this procedure is illustrated in Figure 1, which shows that profiles obtained at approximately the same epoch (within 2 months of each other) using different telescopes match each other very well after the above normalization. This result also shows that the small differences in apertures of the different spectra do not significantly affect the normalization.

The Keck spectra are the only exceptions, as we had to "renormalize" them to $\sim 80 \%$ of the resulting flux after the

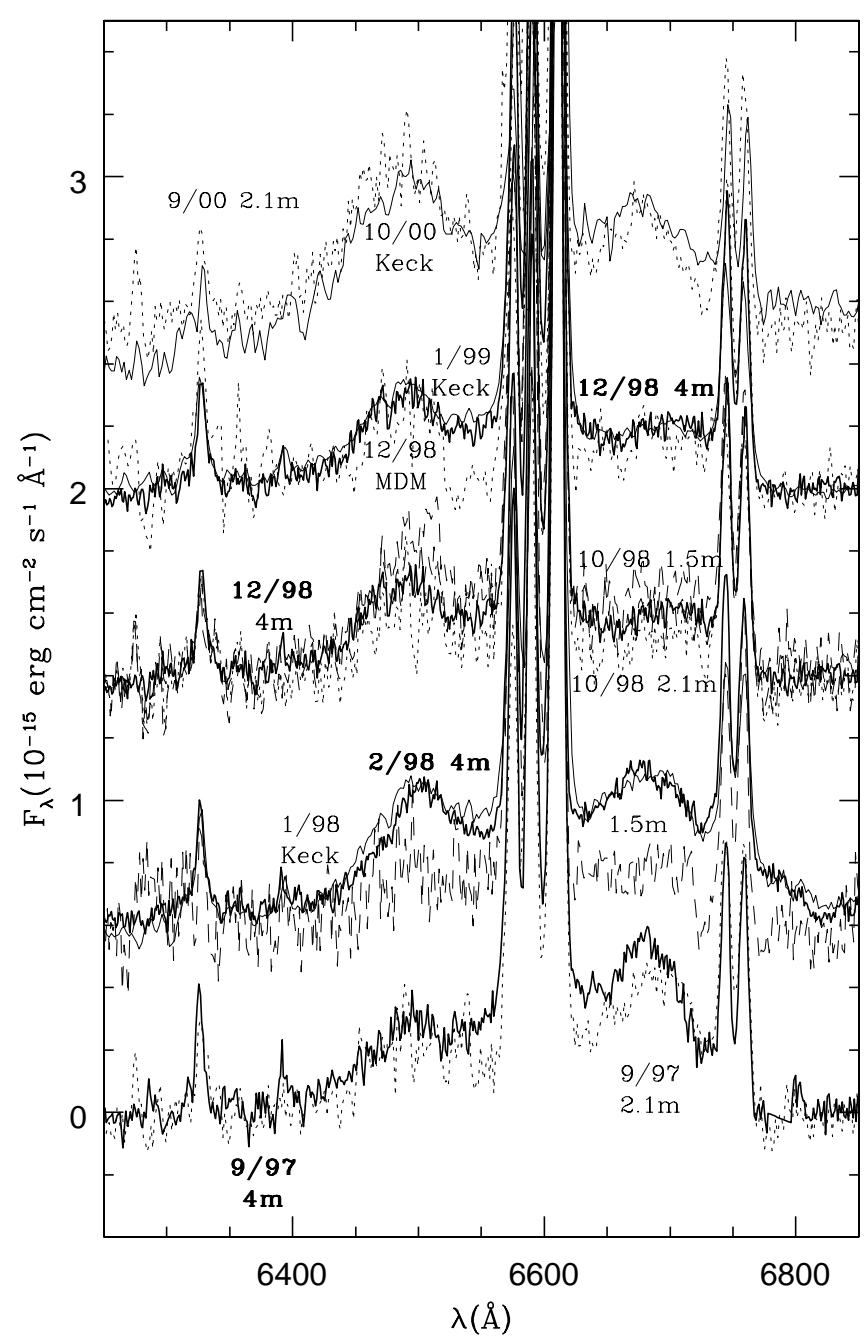

FIG. 1.-Comparison between profiles obtained at approximately the same epoch (within 2 months) but using different telescopes. Spectra are identified in the figure by the epoch (mm/yy) and telescope: CTIO $4 \mathrm{~m}$ (heavy solid line), Keck II (solid line), KPNO 2.1 m (dotted line), MDM for epoch 12/98 (dotted line), and CTIO $1.5 \mathrm{~m}$ (dashed line). normalization described above, to allow an agreement with the spectra obtained at approximately the same epoch with the other large telescopes. We attribute this result to the narrower slit used in the Keck spectra $\left(1^{\prime \prime}\right)$, suggesting that the narrow-line emission extends beyond $1^{\prime \prime}$, although not much more, as the normalization works for the larger apertures. The scaling factors listed in the last column of Table 1 for the Keck spectra are already corrected for this additional normalization.

After normalization, there is reasonable agreement between the CTIO $4 \mathrm{~m}$ and KPNO $2.1 \mathrm{~m}$ spectra of 1997 September, as Figure 1 shows. There is excellent agreement between the spectra with the best signal-to-noise ratios: Keck (thin solid lines; epochs 1998 January and 1999 January) and CTIO $4 \mathrm{~m}$ (thick solid lines; epochs 1998 February and 1998 December). In comparison, the MDM spectrum of 1998 December shows a broad dip around $6530 \AA$ that is not present in the Keck and CTIO spectra. There is also good agreement between the Keck spectra of 2000 October and the KPNO $2.1 \mathrm{~m}$ spectra of 2000 September. The spectra obtained with the CTIO $1.5 \mathrm{~m}$ have the poorest signal-to-noise ratios: the profile from 1998 January has very little flux and is not compatible with the profiles measured with both the Keck and CTIO $4 \mathrm{~m}$ at approximately the same epoch. On the other hand, the $1.5 \mathrm{~m}$ profile of 1998 October agrees with those of the KPNO (1998 October) and the CTIO $4 \mathrm{~m}$ (1998 December).

On the basis of Figure 1, we conclude that we should give more weight in our analysis to the results based on the Keck, CTIO $4 \mathrm{~m}$, and NTT $3.6 \mathrm{~m}$ spectra than to those obtained with the smaller telescopes. This figure also illustrates that changes in the shape of the double-peaked profiles seem to occur on timescales longer than a few $(\sim 3-4)$ months.

\section{RESULTS}

In Figures 2 and 3, we show a sequence of selected $\mathrm{H} \alpha$ profiles from 1991 November 2 to 2002 October 10. Our criterion for the selection was to obtain the most complete time coverage with the available data, using spectra having the highest signal-to-noise ratios in cases of multiple spectra at a given epoch, as discussed above.

During the evolution of the double-peaked $\mathrm{H} \alpha$ profile shown in Figures 2 and 3, the most noticeable changes occur in the relative strengths of the blue and red peaks. The profile starts with the red peak stronger than the blue in 1991 November and evolves to similar strengths of the two peaks in 1994 January, then to the blue peak stronger than the red in 1996 January. The spectrum of 1997 September again shows the red peak stronger than the blue; the two peaks show similar strengths by mid-1998, and then the blue peak is stronger than the red by 1999. In the observation of 2001 September, the blue and red peaks have similar strengths, and in 2002 October the blue peak is stronger again. Another important change that could only be noticed after accumulating data over so many years is a decrease of the broad-line flux at the same time as the blue and red peaks are moving progressively farther apart, as indicated by the measurements below.

In order to quantify the observed variations, we measured the wavelengths of the blue and red peaks, their peak flux densities, and the flux of the broad line, which we list in Table 2. The wavelengths of the blue and red peaks $\left(\lambda_{B}\right.$ and $\left.\lambda_{R}\right)$ and the corresponding peak flux densities $\left(F_{B}\right.$ and $\left.F_{R}\right)$ 


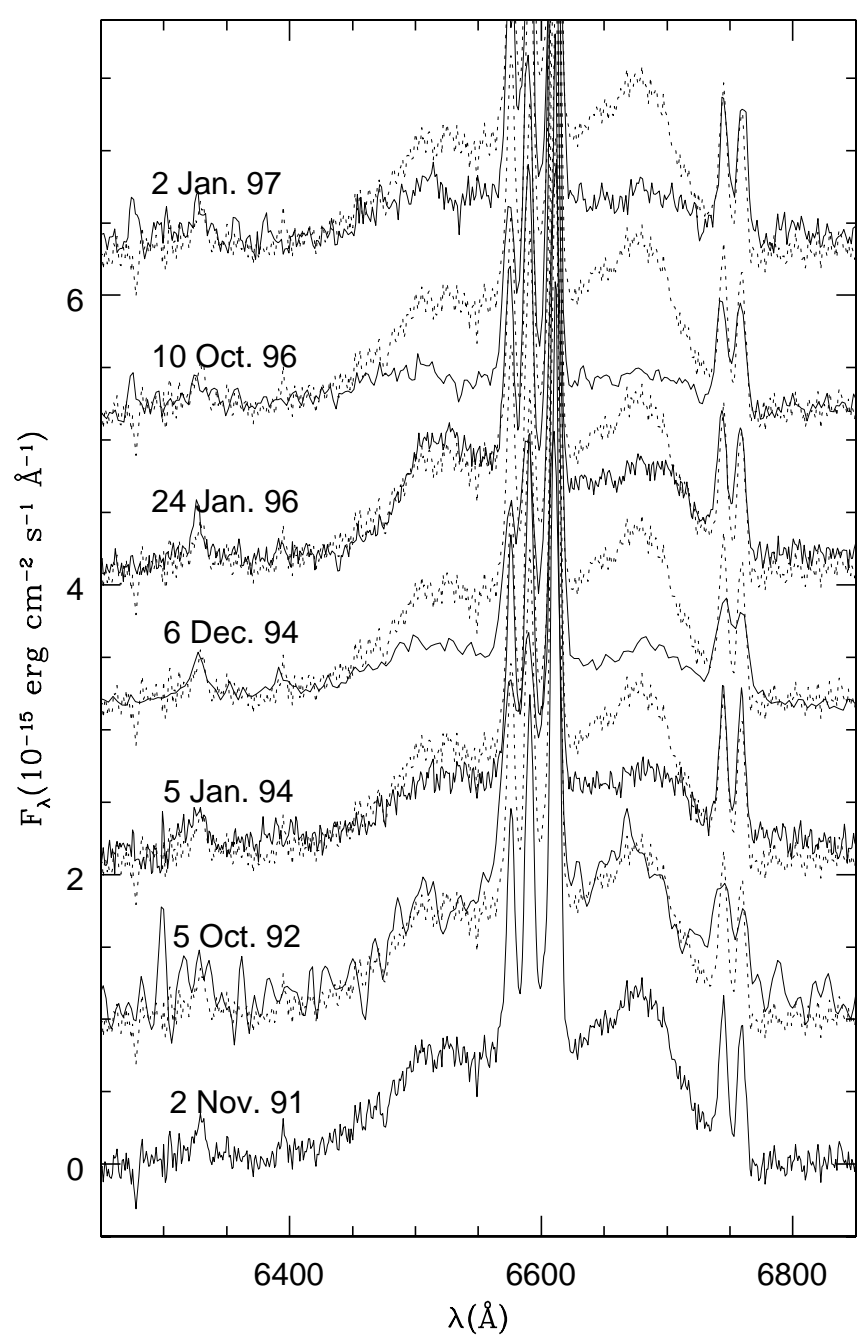

Fig. 2.-Broad, double-peaked $\mathrm{H} \alpha$ profiles from 1991 November to 1997 January (after subtraction of the stellar population contribution). Dotted lines show the 1991 November profile, superposed on the subsequent profiles for comparison.

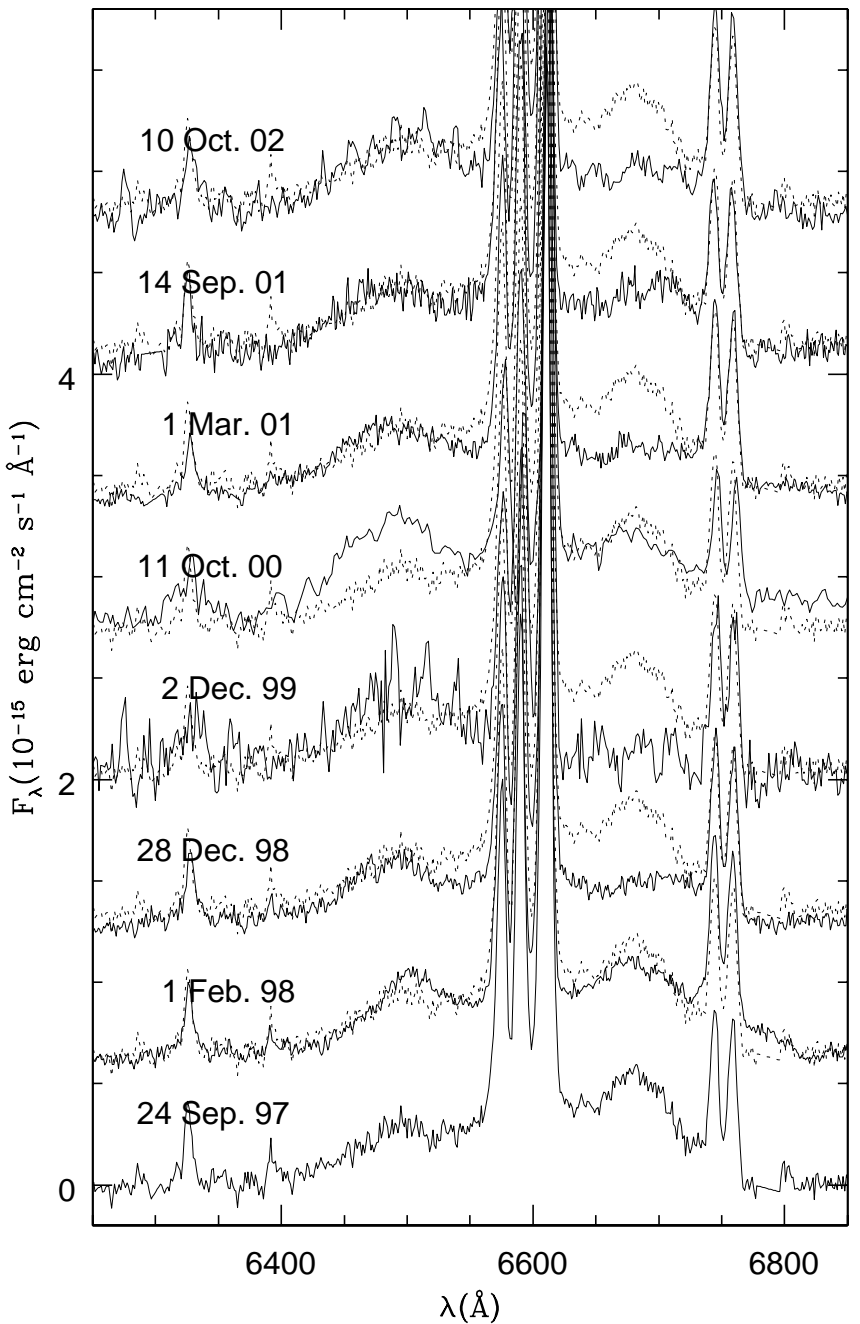

Fig. 3.-Broad, double-peaked $\mathrm{H} \alpha$ profiles from 1997 September to 2002 October (after subtraction of the stellar population contribution). Dotted lines show the 1997 September spectrum, superposed on the subsequent profiles for comparison.

TABLE 2

Measured Properties

\begin{tabular}{|c|c|c|c|c|c|}
\hline Epoch & $\begin{array}{l}\lambda_{B} \\
(\AA)\end{array}$ & $\begin{array}{l}\lambda_{R} \\
(\AA)\end{array}$ & $\begin{array}{c}F_{B}^{\mathrm{a}} \\
\left(10^{-15} \mathrm{ergs} \mathrm{cm}^{-2} \mathrm{~s}^{-1} \AA^{-1}\right)\end{array}$ & $\begin{array}{c}F_{R}^{\mathrm{a}} \\
\left(10^{-15} \mathrm{ergs} \mathrm{cm}^{-2} \mathrm{~s}^{-1} \AA^{-1}\right)\end{array}$ & $\begin{array}{c}F_{\text {broad }}{ }^{\mathrm{b}} \\
\left(10^{-15} \mathrm{ergs} \mathrm{cm}^{-2} \mathrm{~s}^{-1}\right)\end{array}$ \\
\hline $1991.84 \ldots \ldots \ldots \ldots . . .$. & $6528.0 \pm 3.0$ & $6670.4 \pm 1.9$ & $0.754 \pm 0.020$ & $1.172 \pm 0.020$ & $227.0 \pm 9.6$ \\
\hline $1992.76 \ldots \ldots \ldots \ldots . . . .$. & $6535.9 \pm 2.4$ & $6664.6 \pm 2.7$ & $0.801 \pm 0.030$ & $1.122 \pm 0.003$ & $204.3 \pm 13.1$ \\
\hline 1994.01 .............. & $6530.9 \pm 0.4$ & $6671.1 \pm 2.2$ & $0.497 \pm 0.010$ & $0.564 \pm 0.036$ & $120.3 \pm 6.1$ \\
\hline 1994.92 ............... & $6519.3 \pm 2.2$ & $6676.8 \pm 2.2$ & $0.429 \pm 0.024$ & $0.368 \pm 0.032$ & $101.0 \pm 14.0$ \\
\hline 1996.06 .................. & $6528.1 \pm 0.5$ & $6672.3 \pm 3.3$ & $0.854 \pm 0.002$ & $0.628 \pm 0.020$ & $165.7 \pm 3.1$ \\
\hline $1997.73 \ldots \ldots \ldots . . .$. & $6508.6 \pm 5.5$ & $6675.7 \pm 2.7$ & $0.304 \pm 0.017$ & $0.545 \pm 0.016$ & $102.1 \pm 3.8$ \\
\hline 1998.07 .............. & $6504.7 \pm 4.4$ & $6679.3 \pm 1.0$ & $0.398 \pm 0.005$ & $0.430 \pm 0.006$ & $102.3 \pm 11.1$ \\
\hline 1998.09 ................ & $6510.1 \pm 2.5$ & $6679.4 \pm 0.7$ & $0.335 \pm 0.017$ & $0.461 \pm 0.007$ & $104.8 \pm 1.8$ \\
\hline $1998.80 \ldots \ldots \ldots \ldots$ & $6502.1 \pm 2.5$ & $6686.5 \pm 6.5$ & $0.334 \pm 0.015$ & $0.292 \pm 0.012$ & $76.6 \pm 6.0$ \\
\hline 1998.99 ............. & $6496.6 \pm 2.3$ & $6690.5 \pm 5.8$ & $0.283 \pm 0.009$ & $0.225 \pm 0.002$ & $70.1 \pm 2.1$ \\
\hline 1999.02 ................ & $6494.5 \pm 2.2$ & $6687.2 \pm 7.5$ & $0.302 \pm 0.010$ & $0.227 \pm 0.006$ & $71.8 \pm 12.0$ \\
\hline $2000.73 \ldots \ldots \ldots \ldots$ & $6491.8 \pm 2.5$ & $6673.5 \pm 4.0$ & $0.502 \pm 0.020$ & $0.380 \pm 0.015$ & $102.3 \pm 11.0$ \\
\hline 2000.79 ............... & $6493.1 \pm 2.3$ & $6674.4 \pm 3.2$ & $0.441 \pm 0.021$ & $0.298 \pm 0.013$ & $100.0 \pm 7.5$ \\
\hline $2001.16 \ldots \ldots \ldots \ldots . . . . .$. & $6495.1 \pm 1.6$ & $6687.9 \pm 2.8$ & $0.338 \pm 0.001$ & $0.254 \pm 0.005$ & $80.2 \pm 6.8$ \\
\hline $2001.70 \ldots \ldots \ldots \ldots$ & $6496.4 \pm 2.0$ & $6691.4 \pm 1.1$ & $0.326 \pm 0.007$ & $0.338 \pm 0.012$ & $84.4 \pm 8.5$ \\
\hline
\end{tabular}

a Peak flux density.

b Integrated flux. 

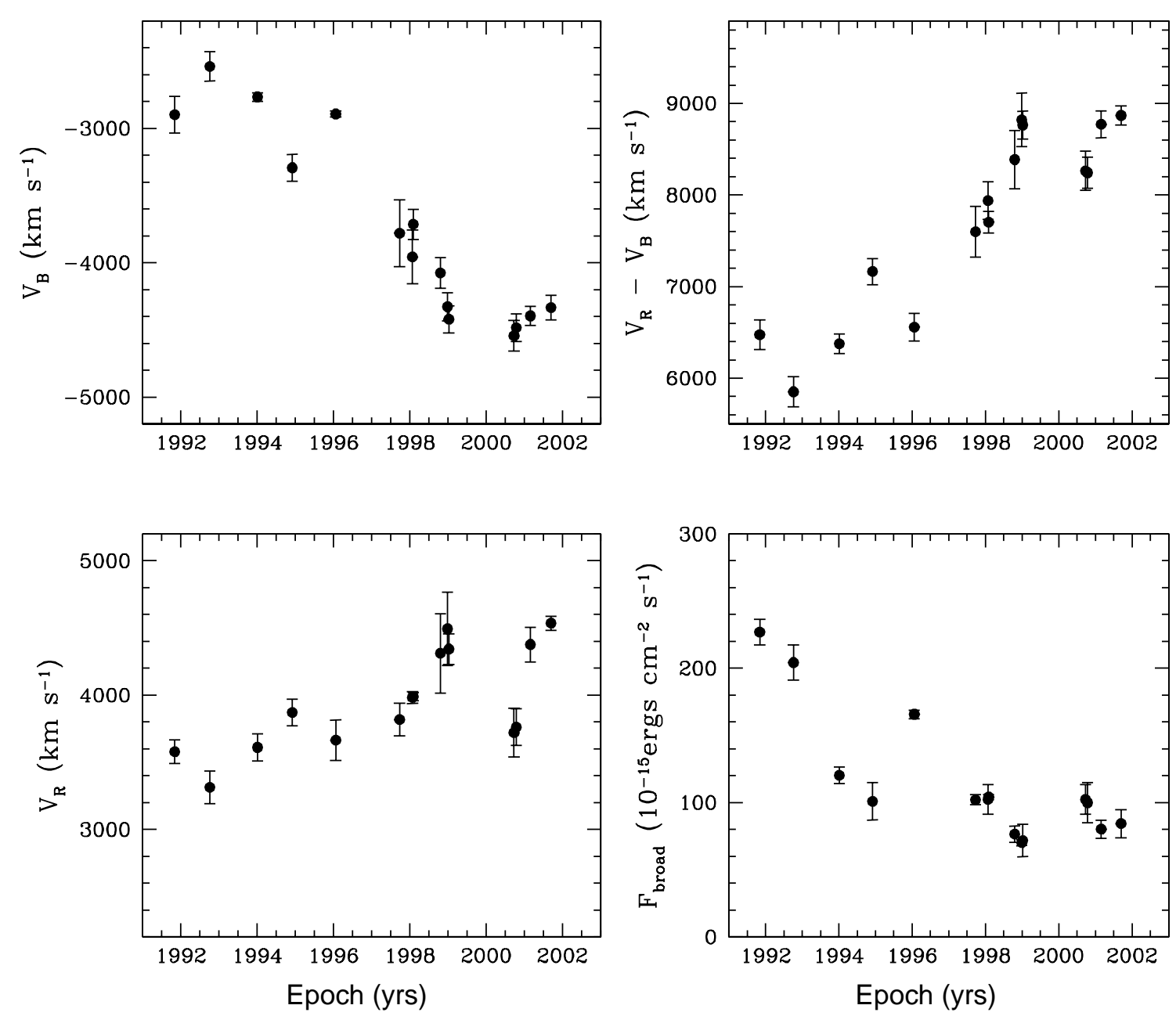

FIG. 4.-Evolution of the measured properties of the double-peaked profiles. Left: Peak velocities corresponding to Gaussian curves fitted to the blue $\left(V_{B}\right.$, top $)$ and red $\left(V_{R}\right.$, bottom $)$ peaks. Right: $V_{R}-V_{B}(t o p)$ and $F_{\text {broad }}$ (bottom).

were obtained by fitting Gaussians separately to the blue and red peaks of the profiles and measuring the central wavelengths and peak fluxes. These measurements were only possible in the large-telescope spectra, as the fits to the blue and red peaks did not consistently converge to a unique central wavelength in spectra with low signal-to-noise ratios. The flux of the broad line $\left(F_{\text {broad }}\right)$ was measured by integrating the fluxes of the broad and narrow emission lines and then subtracting the fluxes of the narrow lines. Uncertainties in the wavelengths and fluxes were estimated by repeating the fits several times after changing by a few angstroms the limits of the windows used to fit the Gaussians and measure the broad-line flux. The resulting average uncertainties are $\sim 5 \AA$ for $\lambda_{B}$ and $\lambda_{R}, \sim 5 \times 10^{-17}$ ergs $\mathrm{cm}^{-2} \mathrm{~s}^{-1} \AA^{-1}$ for $F_{B}$ and $F_{R}$, and $\sim 2 \times 10^{-14} \mathrm{ergs} \mathrm{cm}^{-2}$ $\mathrm{s}^{-1}$ for $F_{\text {broad }}$.

Figure 4 shows the temporal variations of the blue and red peak velocities $\left(V_{B}\right.$ and $\left.V_{R}\right)$, obtained from $\lambda_{B}, \lambda_{R}$, and the average wavelength of the narrow $\mathrm{H} \alpha$ line used as reference $\left(6591.7 \AA\right.$ ), as well as the temporal variation of $V_{R}-V_{B}$ and $F_{\text {broad. }}$. We observe a blueshift of the blue peak from about $-2800 \mathrm{~km} \mathrm{~s}^{-1}$ in the first years to about $-4500 \mathrm{~km} \mathrm{~s}^{-1}$ in 2001, and a redshift of the red peak from $\sim 3500$ to $\sim 4500$ $\mathrm{km} \mathrm{s}^{-1}$ over the same period. The resulting $V_{R}-V_{B}$ increases from $\sim 6000$ to $\sim 9000 \mathrm{~km} \mathrm{~s}^{-1}$. In Figure 5 we show the temporal variation of $F_{B} / F_{R}$.

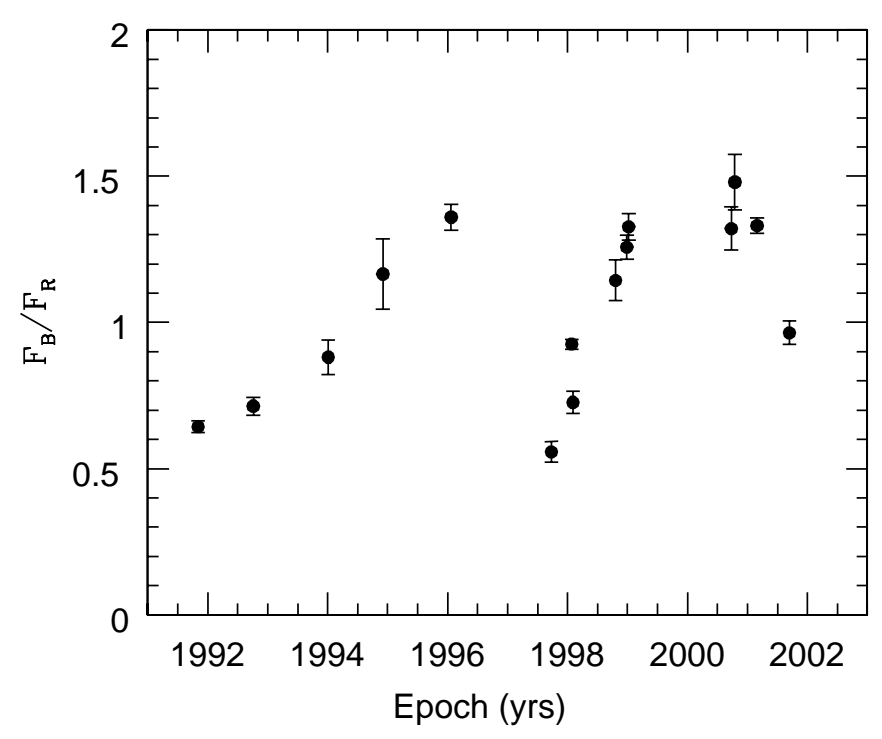

FIG. 5.-Evolution of the ratio between the blue and red peak fluxes (of the fitted Gaussians). 


\section{INTERPRETATION AND COMPARISON WITH MODELS}

\subsection{Preliminary Inferences from the Data}

The continued variation of $F_{B} / F_{R}$ and, in particular, the strong red peak observed in 1997 September still support the origin of the profile in an accretion disk that is not axisymmetric. The asymmetry could be the result of an eccentricity (see, e.g., Eracleous et al. 1995; SB95) or of a circular disk with a disturbance, such as a bright spot (Newman et al. 1997; Zheng, Veilleux, \& Grandi 1991) or spiral arm (see, e.g., Chakrabarti \& Wiita 1993; Gilbert et al. 1999). The precession of the elliptical disk or of the disturbance in the circular disk would be the origin of the variation of $F_{B} / F_{R}$ with time.

The increasing separation of the blue and red peaks $(\S 3)$ indicates that the bulk of the broad-line emission is coming from regions of successively larger velocities. In the accretion disk scenario, this means that the bulk of the emission is coming from successively smaller radii. The above result could suggest at first glance that we are witnessing the infall of matter toward the SMBH. Unfortunately, this seems not to be possible over 10 years of observations. A noticeable decrease in radius should occur in the viscous timescale, which should be larger than $10^{4} \mathrm{yr}$ in the case of NGC 1097 (Frank, King, \& Raine 1992, p. 99, using the black hole mass obtained in $\S 5.2$ ).

An alternative possibility is borne out by the observation that at the same time as the profile is getting broader, the line flux is decreasing (in 2001 September, the broad $\mathrm{H} \alpha$ flux is $\sim \frac{1}{3}$ of the flux of 1991 November), which suggests that the ionizing source is getting dimmer. Thus, a more plausible interpretation is that the dimming of the ionizing source is causing the region of maximum emission to come closer to the center, to regions of larger velocities, and is thus giving rise to broader profiles.

Inspection of Figure 4 shows that there seems indeed to be an inverse correlation between $V_{R}-V_{B}$ and $F_{\text {broad, }}$, not only as a general trend along the 11 years covered by the observations, but also from epoch to epoch. For example, in 1996 January the flux of the line showed an increase relative to the two previous epochs, while $V_{R}-V_{B}$ decreased. The same seems to have happened in 2000 September-October. This inverse correlation can be better observed in Figure 6, where we plot $V_{R}-V_{B}$ against $F_{\text {broad. }}$.

The above effect (the inverse correlation between the ionizing flux and the width of the profile) has been previously discussed by Dumont \& Collin-Souffrin (1990). These authors explain the existence of a "radius for maximum emission" or "saturation radius" as follows. In the lineemitting region of the accretion disk, there are two effects contributing to the line intensity: the increase of the emitting surface with distance from the center and the decrease of the intensity of ionizing radiation with radius. The ionizing flux can become quite high close to the center, leading to ionization of the disk and saturation of the line emission (cooling is mainly due to free-bound and free-free processes and not to lines). The radius at which this saturation sets in is the radius of maximum emission.

Finally, we note that in Figure 4, the velocity of the blue peak shows a steeper variation (blueshift) than that of the red peak (redshift), implying a net blueshift of the center between the two peaks of about $-450 \mathrm{~km} \mathrm{~s}^{-1}$ between 1991 November and 2001 September. One possibility is that this

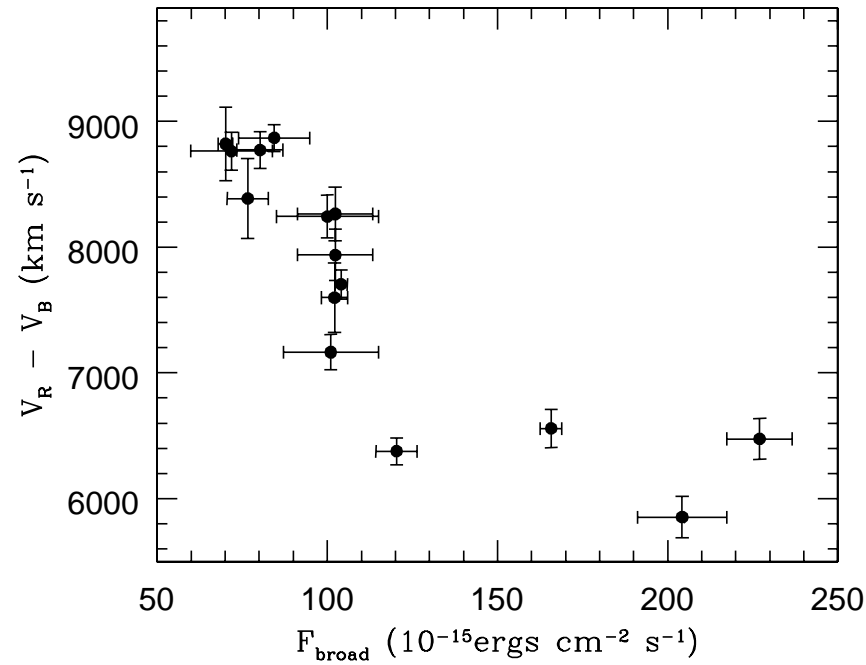

FIG. 6.-Inverse correlation between the velocity difference between the two peaks and the flux of the broad $\mathrm{H} \alpha$ line.

blueshift is due to a wind originating in the disk, which would be consistent with observations of $\mathrm{Fe}$ II absorption lines in a recent HST spectrum of NGC 1097 (Eracleous 2002) that indicate the presence of an outflow.

With these considerations in mind, we have tried to fit the variable line profiles with two different models, as we describe in detail below. Both models rely on a nonaxisymmetric disk structure and its precession to reproduce the changes in the relative strengths of the two peaks. However, this mechanism cannot explain the increasing separation of the two peaks. The latter effect requires us to change the radius of maximum line emission in the disk by adjusting the prescription for the disk emissivity.

\subsection{Elliptical-Disk Model}

In Eracleous et al. (1995), we showed that an ellipticalaccretion ring model could reproduce the broad, doublepeaked $\mathrm{H} \alpha$ profile of NGC 1097, as observed in 1991 November. After following the variations of the line profile from 1991 to 1996 (SB97), we developed the model further, so that the plane of the ring was inclined at $i=34^{\circ}$ to the line of sight and its line-emitting part corresponded to a range of pericenter distances between $\xi_{1}=1300$ and $\xi_{2}=1600$, where $\xi$ is expressed in units of the gravitational radius $r_{q} \equiv G M / c^{2}$. The ring was circular up to a radius $\xi_{c}=1400$, and from there the eccentricity increased linearly from $e=0$ to 0.45 . The ring had a broadening parameter $\sigma=1200 \mathrm{~km} \mathrm{~s}^{-1}$ and an emissivity varying as $\epsilon(\xi)=\epsilon_{0} \xi^{-q}$. The variation of the observed line profiles from 1991 to 1996 was reproduced by the precession of the ring with a period of $25 \mathrm{yr}$, which led to an estimate of the central black hole mass of $9 \times 10^{5} M_{\odot}$.

We tried to use the same elliptical-ring model to reproduce all the double-peaked $\mathrm{H} \alpha$ profiles observed from 1991 to 2002 , changing only the orientation angle, $\phi_{0}$, under the assumption that the precession continued. However, we were not able to reproduce the line profiles observed after 1996; continued precession alone could explain the variation in the relative heights of the two peaks but not the observed velocity shifts of the blue and red peaks. After 
exploring the effect of other parameters on the shape of the line profile, we found that only changes in the inner pericenter distance of the ring (from $\xi_{1}=1300$ to 450 ) could lead to the observed increase in the separation of the two peaks, $\lambda_{R}-\lambda_{B}$. Such a dramatic change in the physical location of a given volume of gas could not occur on a timescale of $5 \mathrm{yr}$, however, as we noted in $\S 4.1$. Rather, the emission properties of the ring must have changed, so that the radius of maximum emission became smaller. Therefore, to produce a self-consistent and physically plausible scenario for the entire set of observations, we were led to revise the model parameters. This revision represents a refinement of earlier models, which is possible only after following the variability of the line profile over a baseline of $10 \mathrm{yr}$. We now propose that the ring has always had a smaller inner radius of $\xi_{1}=450$ (and now resembles a disk more than a ring) and that the increasing width of the profile is due to a change in emissivity such that, in more recent epochs, the inner regions of the disk make a more significant relative contribution to the total line emission. This picture is also consistent with the observational result that the flux of the broad line is decreasing, as discussed above.

To explain the increasing separation between the two peaks, we experimented with several emissivity laws. We found that we could best reproduce the observations with a broken power law, such that inside a radius $\xi_{q}$ the powerlaw index is $q_{1}=-1$ and outside this radius $q_{2}=1$. This means that the emissivity increases with radius from $\xi_{1}$ to $\xi_{q}$ and then decreases with radius beyond $\xi_{q}$. These $q$-values are also physically motivated: in the "saturation" region, the increase in emissivity is due to the increase in the area of the ring, while beyond $\xi_{q}$, the decrease can be understood as due to both the drop-off in the intensity of the radiation field as $r^{-2}$ and the increase of the emitting area as $r$.

In this parameterization of the disk emissivity, varying the "break radius" $\xi_{q}$ allows us to reproduce the shifts of the blue and red peaks. In particular, allowing the break radius to decrease with time shifts the region of maximum emission closer to the center and results in an increase in the separation between the two peaks. At the same time, since the relative heights of the two peaks have continued to change, we allow the disk to precess (as in SB97).

To fit individual line profiles, we fixed the inner and outer pericenter distances of the line-emitting disk to $\xi_{1}=450$ and $\xi_{2}=1600$ (respectively), the inclination angle to $i=34^{\circ}$, and the broadening parameter to $\sigma=1200 \mathrm{~km} \mathrm{~s}^{-1}$. The eccentricity was allowed to increase linearly with radius, from $e\left(\xi_{1}\right)=0$ to $e\left(\xi_{2}\right)=0.45$. The emissivity of the disk $\left(\epsilon \propto \xi^{-q}\right)$ was set to increase with radius in the inner disk (i.e., $q_{1}=-1$ up to a radius $\xi_{q}$ ) and then decrease $\left(q_{2}=1\right)$. The orientation angle of the disk, $\phi_{0}$, and the emissivity break radius, $\xi_{q}$, were the only parameters allowed to vary to represent a precession of the disk and a decline in the illumination. We first searched for models that could reproduce the primary observables, namely, the velocities and relative strengths of the two peaks. The best-fitting models resulting from the above procedure are compared with the observations in Figures 7 and 8, and the model parameters are summarized in Table 3.

In the last epochs, beginning in 1998 December, the fits of the model to the data show some improvement if we allow a small blueshift of the central wavelength of the profile $(\sim 10$ $\AA$ ) relative to the wavelength of the narrow $\mathrm{H} \alpha$ line $(6591.7$ $\AA$ - which we have so far adopted as the center of the broad

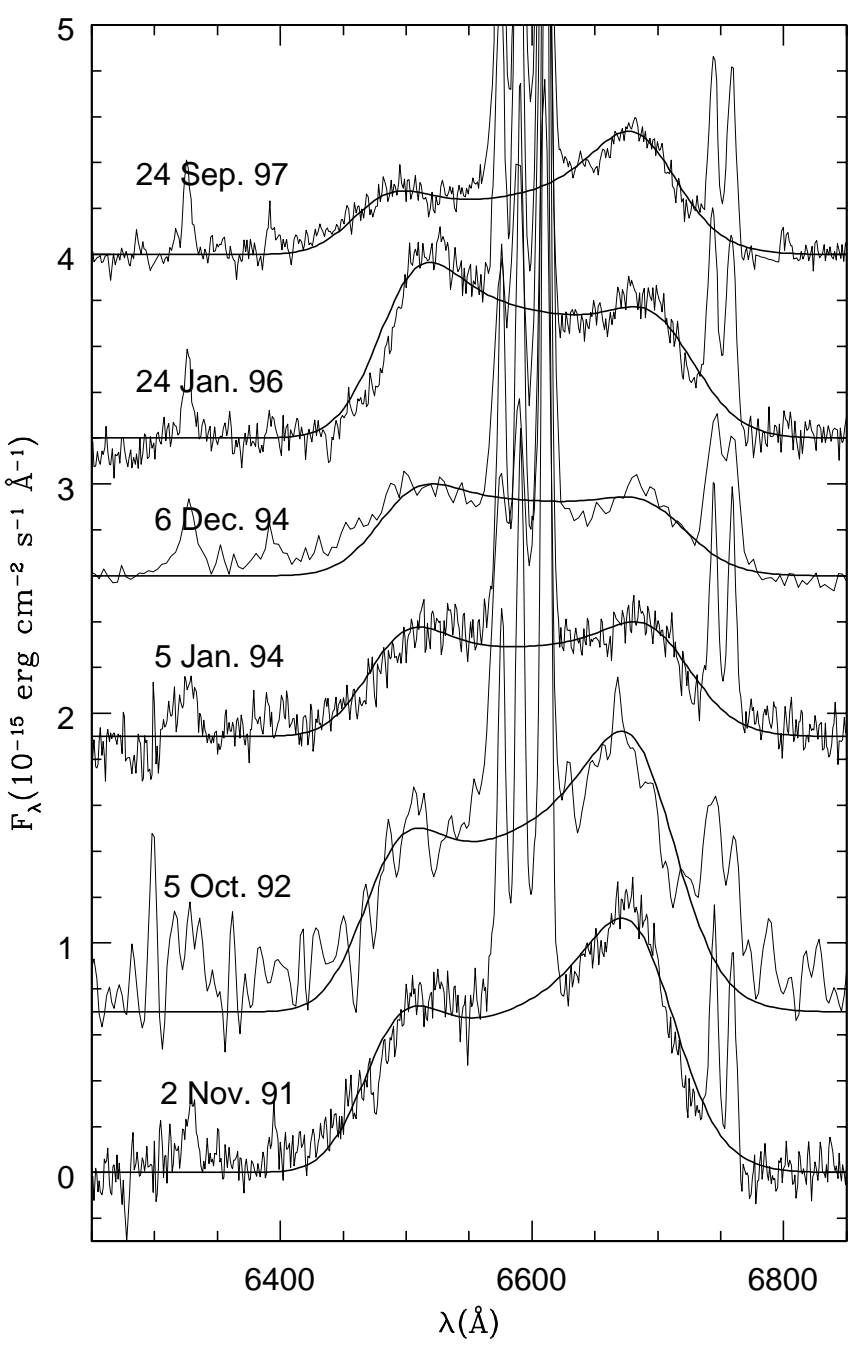

FIG. 7.-Fits of the elliptical-disk model to the profiles from 1991 November to 1997 September obtained by changing only $\phi_{0}$ (the orientation of the disk relative to the line of sight) and $\xi_{q}$ (the radius of the peak of the emissivity law). The values of these parameters are listed in Table 3 .

line in the models). We show these improved fits as dotted lines in Figure 8. This blueshift of the broad line was already detected in the observed evolution of the blue and red peak velocities (Fig. 4) and was discussed at the end of $\S 4.1$.

TABLE 3

Elliptical-Disk Model PARAMETERS

\begin{tabular}{crr}
\hline \hline Epoch & $\xi_{q}$ & $\begin{array}{c}\phi_{0} \\
(\mathrm{deg})\end{array}$ \\
\hline $1991.84 \ldots \ldots \ldots \ldots$. & 1260 & 152 \\
$1992.76 \ldots \ldots \ldots \ldots$. & 1260 & 152 \\
$1994.01 \ldots \ldots \ldots \ldots$. & 1200 & 171 \\
$1994.92 \ldots \ldots \ldots \ldots$. & 1310 & 182 \\
$1996.06 \ldots \ldots \ldots \ldots$. & 1260 & 189 \\
$1997.73 \ldots \ldots \ldots \ldots$. & 1140 & 131 \\
$1998.09 \ldots \ldots \ldots \ldots$. & 1110 & 164 \\
$1998.99 \ldots \ldots \ldots \ldots$. & 940 & 202 \\
$2000.79 \ldots \ldots \ldots \ldots$. & 950 & 187 \\
$2001.16 \ldots \ldots \ldots \ldots$. & 798 & 209 \\
$2001.70 \ldots \ldots \ldots \ldots$. & 960 & 168 \\
\hline
\end{tabular}




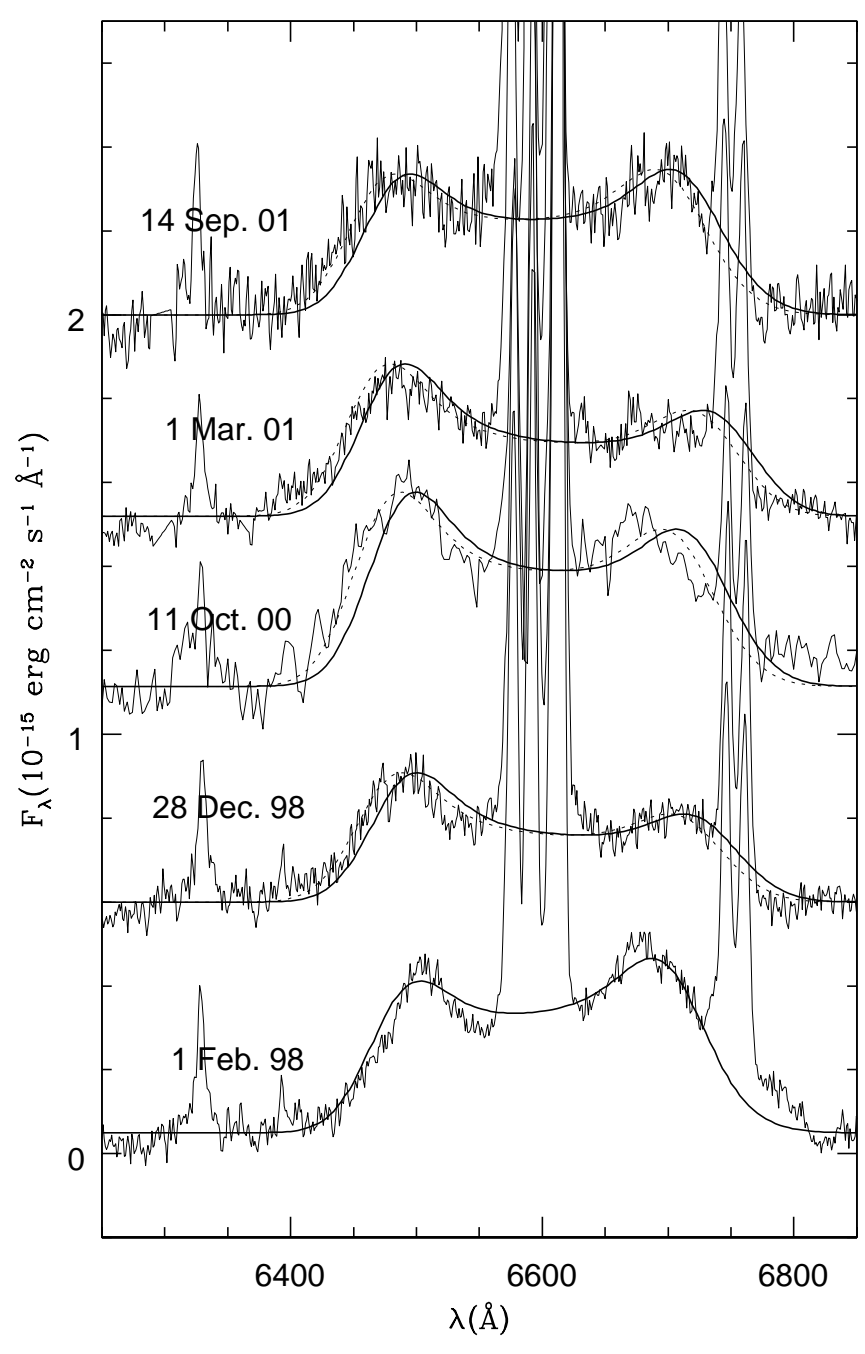

FIg. 8.-Same as Fig. 7, but for 1998 February-2001 September. Dotted lines show these fits blueshifted by $12 \AA$.

The evolution of $\xi_{q}$ and $\phi_{0}$ is shown in Figure 9. There is very little change in $\xi_{q}$ until 1996, but then in 1997 it begins to decrease, causing the relative contribution of the inner parts of the disk to become more important and producing the broadening of the profile. The orientation angle of the disk, represented by $\phi_{0}$, shows a small range of values, $131^{\circ}<\phi_{0}<209^{\circ}$, as listed in Table 3 . Noticing that changes in orientation by half a revolution result in very similar (although not identical) model profiles, we have exploited this property in order to construct a series of models that would approximately represent a precession of the disk. The best compromise we could obtain between a good fit of the profiles and the precession scenario is illustrated in the bottom panel of Figure 9. Assuming that the precession rate of the disk is constant, we carry out a linear leastsquares fit to the precession phase, shown in Figure 9. We adopt a functional form given by

$$
\frac{\phi_{0}}{2 \pi}=\frac{t-t_{0}}{P_{\text {prec }}},
$$

where $t$ is the time in years, $t_{0}$ is an arbitrary zero point of the precession cycle, and $P_{\text {prec }}$ is the precession period. The best fit, shown as a solid line in Figure 9, yields $t_{0}=1990.8$

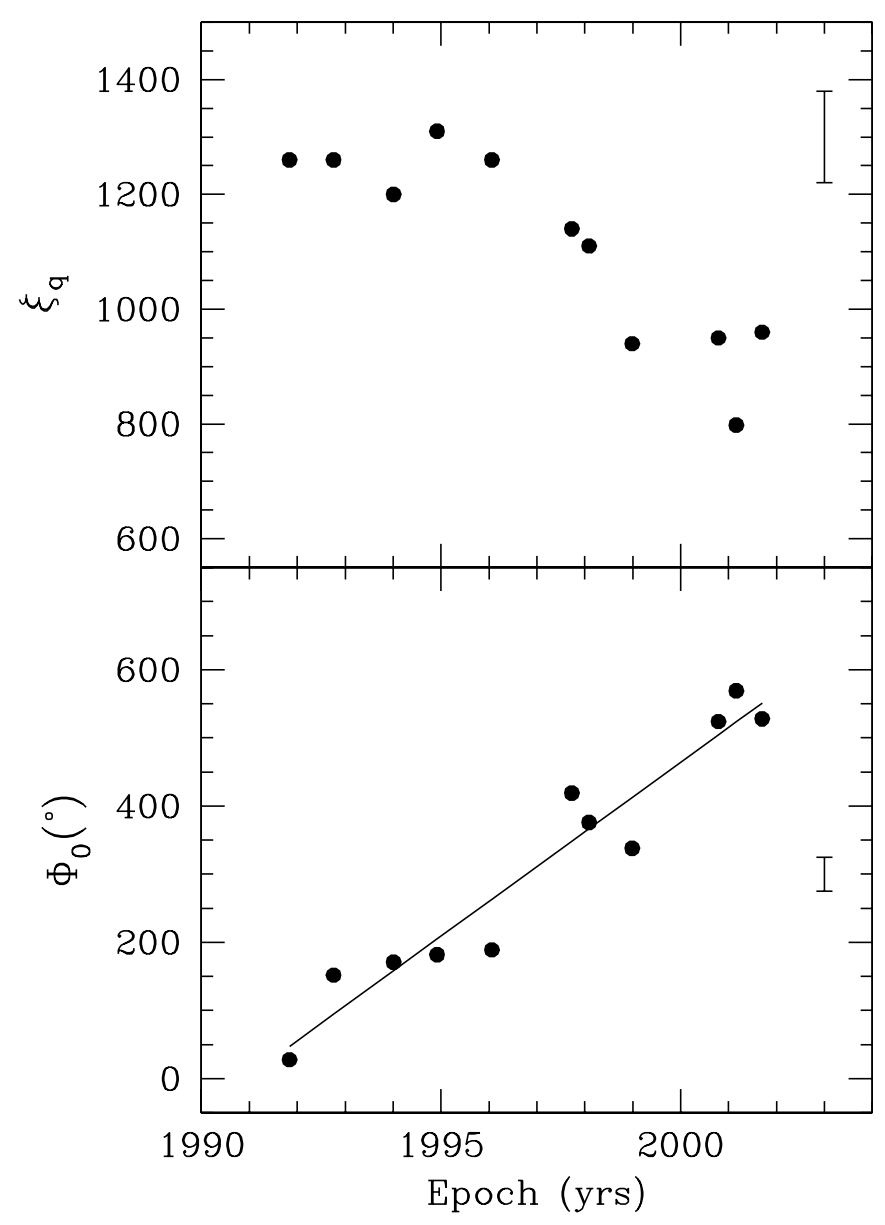

FIG. 9.-Evolution of the elliptical-disk model parameters: emissivitylaw peak radius $\xi_{q}$ (top) and orientation of the disk relative to the line of sight $\phi_{0}$ (bottom).

and $P_{\mathrm{prec}}=7.1 \mathrm{yr}$, which implies that the disk has completed almost two revolutions since the epoch of the first observation, as one would expect from the behavior of the peak strength ratio, $F_{B} / F_{R}$, shown in Figure 5. In Figure 10, we show a cartoon of the accretion disk in two epochs, the first (1991.8) and last (2001.7) fitted by the model, illustrating the change in the disk emissivity in $10 \mathrm{yr}$.

In conclusion, we note that even though models that represent a smooth precession of the eccentric disk are preferable on physical grounds, they do not provide as good a description of the line profiles as the models whose parameters are listed in Table 3.

\subsection{Circular Disk-plus-Spiral Arm Model}

An alternative to the elliptical-disk model is a model that invokes a nonaxisymmetric perturbation of the emissivity of a circular disk. Models of this type were applied to the variable $\mathrm{H} \alpha$ profile of 3C 390.3 by Zheng et al. (1991; a "bright sector" in the disk) and Gilbert et al. (1999; a single-arm spiral, inspired by the work of Chakrabarti \& Wiita 1993, 1994). An important feature of these models is that the perturbation spans a relatively wide range in radius (and projected velocity), which leads to a modulation in the relative strengths of the two peaks of the line profile without creating a "third peak" that moves across the profile. Spiral-arm models have additional appeal because spiral 

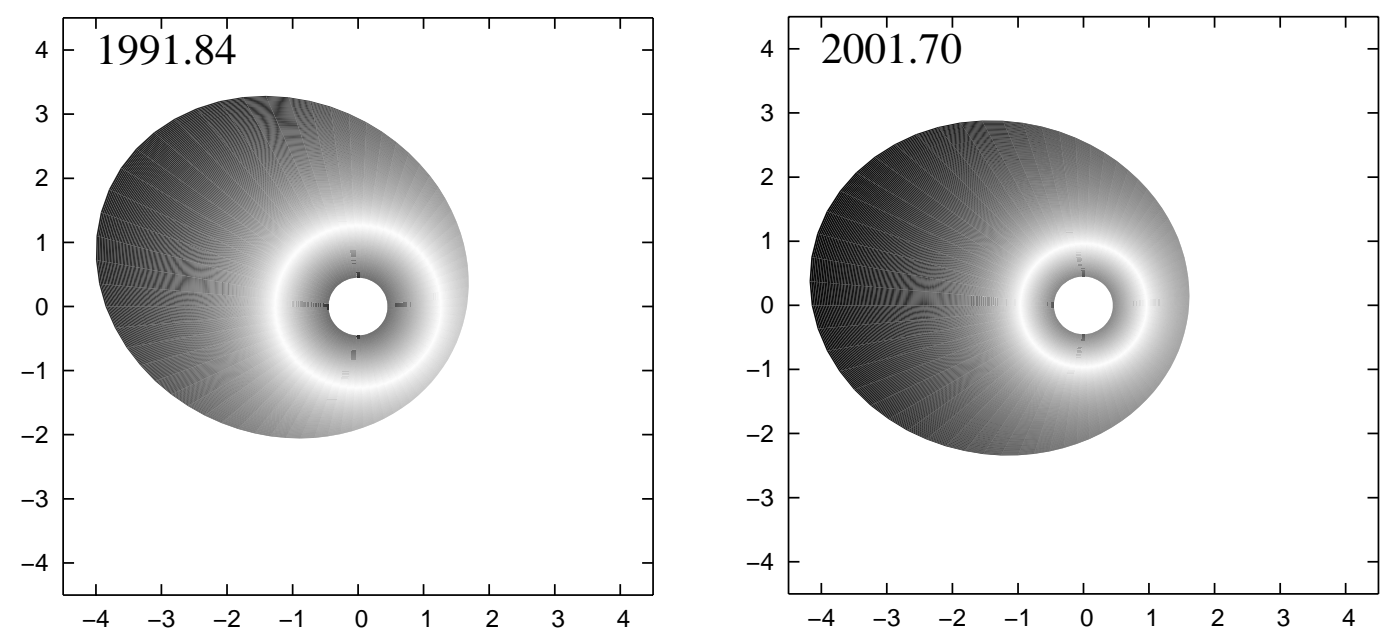

FIG. 10.-Elliptical-disk cartoon, illustrating the change in the disk emissivity and orientation between the first and last epochs fitted with this model. Units are $10^{3} r_{g}$, and the observer is to the right. Emissivity scale is arbitrary, white representing the brightest regions.

arms are known to exist in other astrophysical disks, such as the disks of spiral galaxies and the accretion disks of some cataclysmic variables (see, e.g., Steeghs, Harlaftis, \& Horne 1997; Baptista \& Catalán 2000; Patterson, Halpern, \& Shambrook 1993).

We adopt the formulation of Gilbert et al. (1999), according to which the line-emitting portion of the disk is circular, has an inclination angle $i$, and is bounded by inner and outer radii $\xi_{1}$ and $\xi_{2}$. Superposed on the axisymmetric emissivity pattern $\left(\epsilon \propto \xi^{-q}\right)$ is an emissivity perturbation, which has the form of a single spiral arm. We adopted a single-arm pattern after experimenting with patterns having two or more spiral arms. We found that two or more spiral arms could not reproduce the changing asymmetry of the $\mathrm{H} \alpha$ line profile of NGC 1097, a conclusion also reached by Gilbert et al. (1999) for 3C 390.3.

The prescription for the total emissivity of the disk is given by

$$
\begin{aligned}
\epsilon(\xi, \phi)=\epsilon_{0} \xi^{-q}\{ & 1+\frac{A}{2} \exp \left[-\frac{4 \ln 2}{\delta^{2}}\left(\phi-\psi_{0}\right)^{2}\right] \\
& \left.+\frac{A}{2} \exp \left[-\frac{4 \ln 2}{\delta^{2}}\left(2 \pi-\phi+\psi_{0}\right)^{2}\right]\right\},
\end{aligned}
$$

where the two exponentials represent the decay of the emissivity of the spiral arm with azimuthal distance on either side of the ridge line. The parameter $\delta$ represents the azimuthal width of the spiral arm (FWHM), while the quantity $\phi-\psi_{0}$ is the azimuthal distance from the ridge of the spiral arm, which is defined by

$$
\psi_{0}=\phi_{0}+\frac{\log \left(\xi / \xi_{\mathrm{sp}}\right)}{\tan p},
$$

where $p$ is the pitch angle and $\xi_{\mathrm{sp}}$ is the innermost radius of the spiral arm (also used as a fiducial radius for normalization; the spiral arm is set to begin at the outer radius of the disk, $\xi_{2}$ ). The parameter $A$ represents the brightness contrast between the spiral arm and the underlying, axisymmetric disk, while $\phi_{0}$ sets the azimuthal orientation of the spiral pattern.

To fit individual line profiles, we followed the procedure described in $\S 4.2$. We found that we could keep the inner and outer radii of the disk at the values of the inner and outer pericenter distances of the elliptical-disk model, namely, $\xi_{1}=450$ and $\xi_{2}=1600$. We also kept the inclination at $i=34^{\circ}$ and the broadening parameter at $\sigma=1200$ $\mathrm{km} \mathrm{s}^{-1}$. The only parameters we needed to vary in order to reproduce the evolution of the profiles were $q, A$, and $\phi_{0}$. The remaining spiral-arm parameters were kept at the following values: $\xi_{\mathrm{sp}}=\xi_{1}, p=-50^{\circ}$, and $\delta=70^{\circ}$. As in the case of the elliptical-disk model, the variation of $\phi_{0}$ can explain the modulation of the relative strengths of the two peaks of the $\mathrm{H} \alpha$ line but not the increasing separation between them. Therefore, in the spirit of the discussion of $\S 4.1$, we allowed the emissivity power-law index to vary so as to shift the region of maximum emission to smaller radii.

The best-fitting models are compared to the observed line profiles in Figures 11 and 12, while the corresponding parameter values (for $A, q$, and $\phi_{0}$ ) are listed in Table 4 . The spiral-arm contrast, parameterized by $A$, has decreased from 3 to 2 since the first observations, while $q$ has increased, favoring the emission of the inner part of the disk relative to that of the outer parts in the later epochs and producing the broadening of the profile. The $\phi_{0}$ values were constrained to allow a monotonic precession of the spiral pattern, as illustrated in Figure 13, where we show how $q$ and $\phi_{0}$ evolve with time. The latter figure shows that the

TABLE 4

SPIRAL-Disk Model Parameters

\begin{tabular}{ccrr}
\hline \hline Epoch & $A$ & $q$ & $\begin{array}{c}\phi_{0} \\
(\mathrm{deg})\end{array}$ \\
\hline $1991.84 \ldots \ldots \ldots \ldots$ & 3 & -1.0 & 30 \\
$1992.76 \ldots \ldots \ldots \ldots$. & 3 & -1.0 & 140 \\
$1994.01 \ldots \ldots \ldots \ldots$. & 2 & -1.0 & 170 \\
$1994.92 \ldots \ldots \ldots \ldots$. & 2 & -1.0 & 200 \\
$1996.06 \ldots \ldots \ldots \ldots$. & 2 & -1.0 & 320 \\
$1997.73 \ldots \ldots \ldots \ldots$. & 3 & 0.0 & 420 \\
$1998.09 \ldots \ldots \ldots \ldots$. & 2 & 1.5 & 470 \\
$1998.99 \ldots \ldots \ldots \ldots$. & 2 & 2.3 & 540 \\
$2000.79 \ldots \ldots \ldots \ldots$. & 2 & 2.7 & 600 \\
$2001.16 \ldots \ldots \ldots \ldots$. & 2 & 3.0 & 640 \\
$2001.70 \ldots \ldots \ldots \ldots$. & 2 & 3.0 & 710 \\
\hline
\end{tabular}




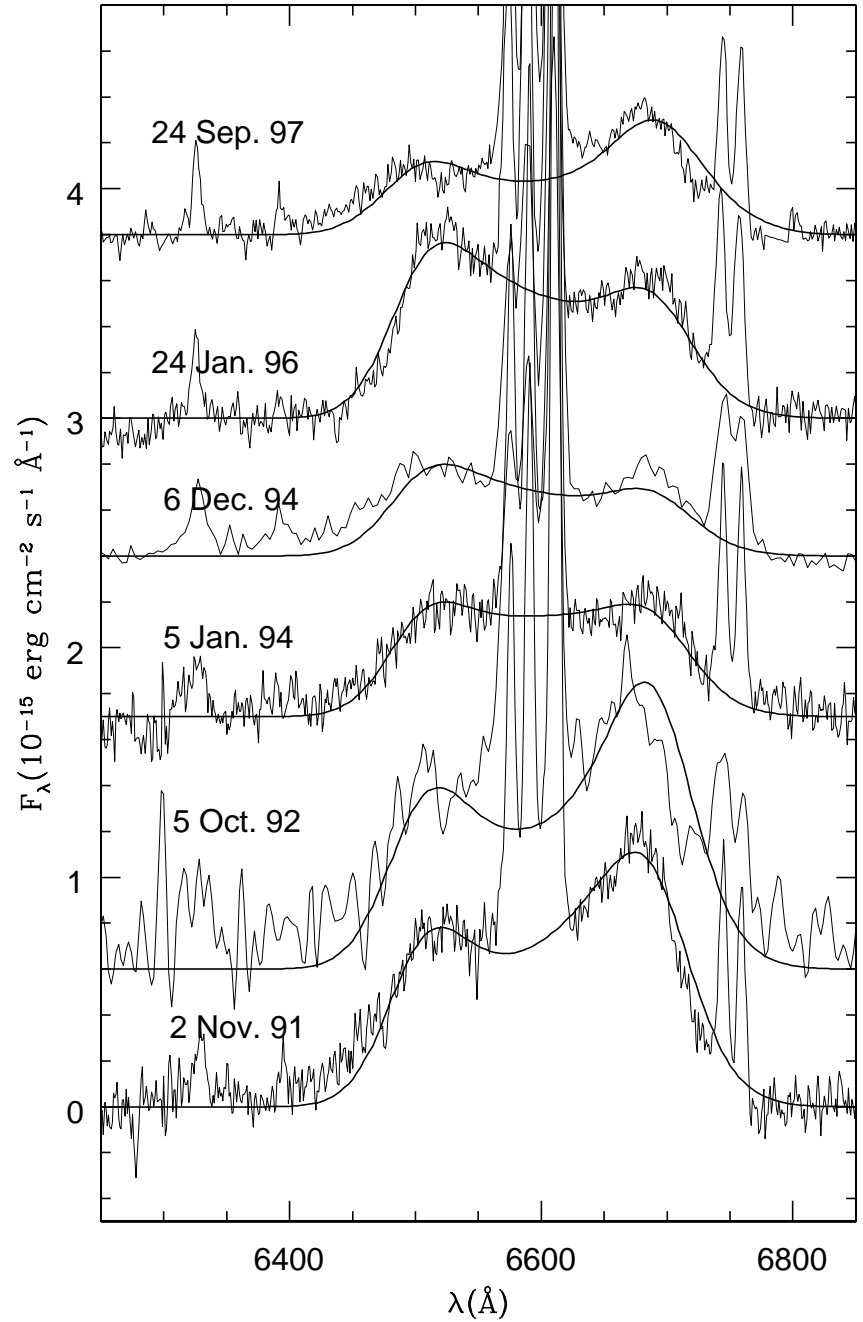

FIG. 11.-Fits of the spiral-disk model to the profiles from 1991 November to 1997 September obtained by changing $\phi_{0}$ (the orientation of the spiral arm relative to the line of sight), $q$ (the slope of the radial component of the emissivity law), and $A$ (the spiral-arm contrast parameter). The values of these parameters are listed in Table 4.

spiral model better accommodates a monotonic precession (of the spiral pattern) than the elliptical model. The solid line in the bottom panel of Figure 13 is a fit to the precession phase following equation (1), yielding $t_{0}=1991.3$ and $P_{\text {prec }}=5.5 \pm 0.3 \mathrm{yr}$. Such a period implies, again, that the disk has completed almost two revolutions since the epoch of the first observation, as one would expect from the behavior of the peak strength ratio $\left(F_{B} / F_{R}\right)$ shown in Figure 5.

In Figure 14 we illustrate a cartoon of the disk in the first and last epochs fitted by the model. Note that the spiral arm is quite "broad" and hard to observe in the last epoch because of the low contrast relative to the disk, as implied by the best-fitting parameters (Table 4 ).

\section{DISCUSSION}

\subsection{Comparison with Studies of Other Objects}

The variation of the relative strengths of the two peaks of the $\mathrm{H} \alpha$ line of NGC 1097 appears to follow the same trend as in broad-line radio galaxies. Examples include 3C 390.3

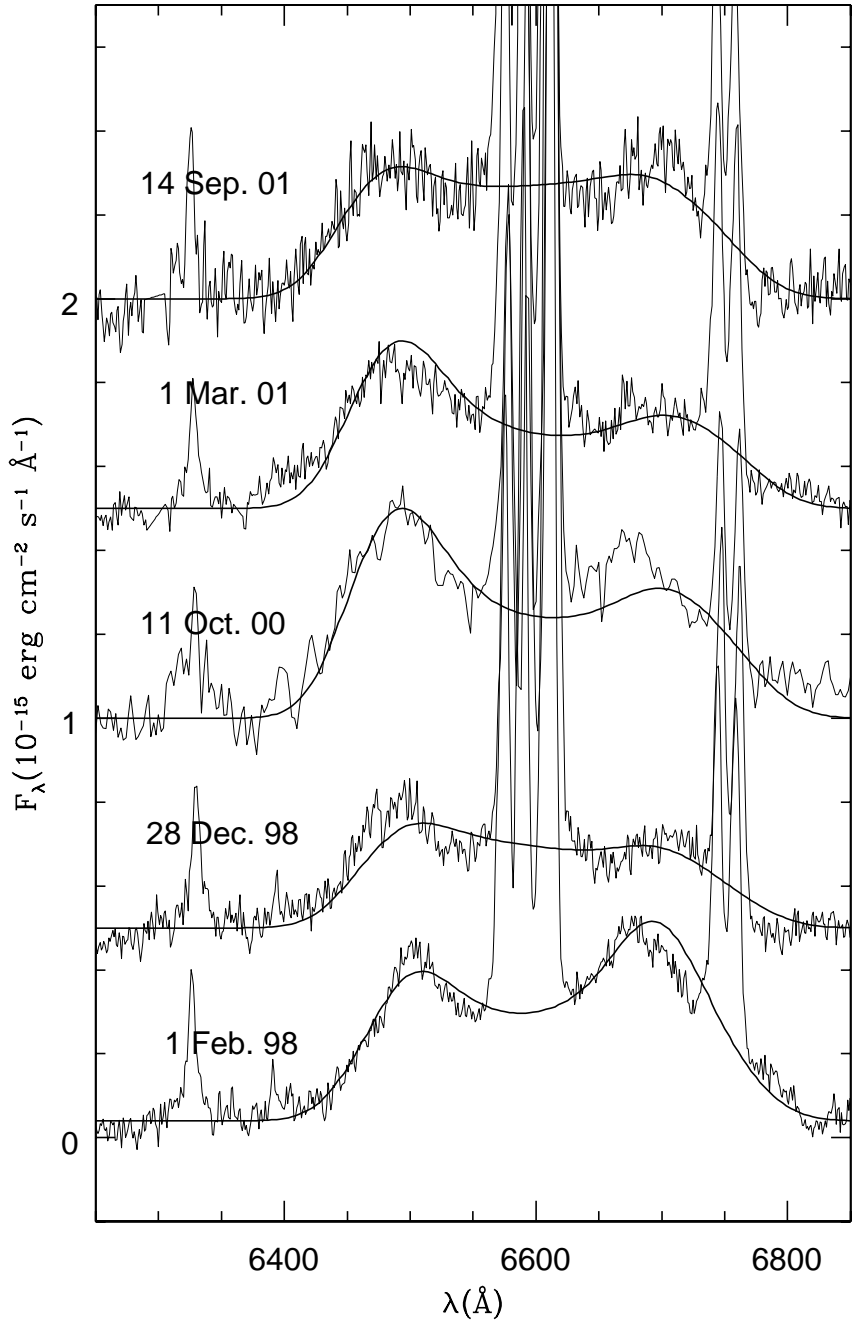

FIG. 12.-Same as Fig. 11, but for 1998 February-2001 September

(Zheng et al. 1991; Gilbert et al. 1999), 3C 332 (Gilbert et al. 1999), Pictor A (Eracleous \& Halpern 1998), and Arp 102B (Newman et al. 1997). On the other hand, drifts in the peak wavelengths of double-peaked profiles are seldom observed. One case previously reported is that of the radio galaxy $3 \mathrm{C}$ 390.3, for which Gaskell (1996), Eracleous et al. (1997), and Gilbert et al. (1999) found a drift in the velocity of the blue peak between 1970 and 1996. However, in 3C 390.3, Eracleous et al. (1997) found that the two peaks moved together, unlike what is observed in the case of NGC 1097 , in which the two peaks move apart from each other.

On the other hand, Shapovalova et al. (2001), using observations of the $\mathrm{H} \beta$ profile of $3 \mathrm{C} 390.3$ from 1995 to 2000 (later than those reported by Eracleous et al. 1997), claim to have found drifts of the two peaks in opposite directions (or velocities), arguing that the difference between the velocities of the blue and red peaks is inversely correlated with the continuum flux. They note that when the $\mathrm{H} \beta$ flux decreases, the radial velocity difference between the red and blue peaks increases, while when the $\mathrm{H} \beta$ flux increases, the velocity difference between the two peaks decreases. We point out, however, that the measurement of the wavelength of the red peak of the $\mathrm{H} \beta$ line in 3 C 390.3 is quite uncertain because this peak is severely contaminated by the [O III] $\lambda \lambda$ 4959, 5007 emission lines. 


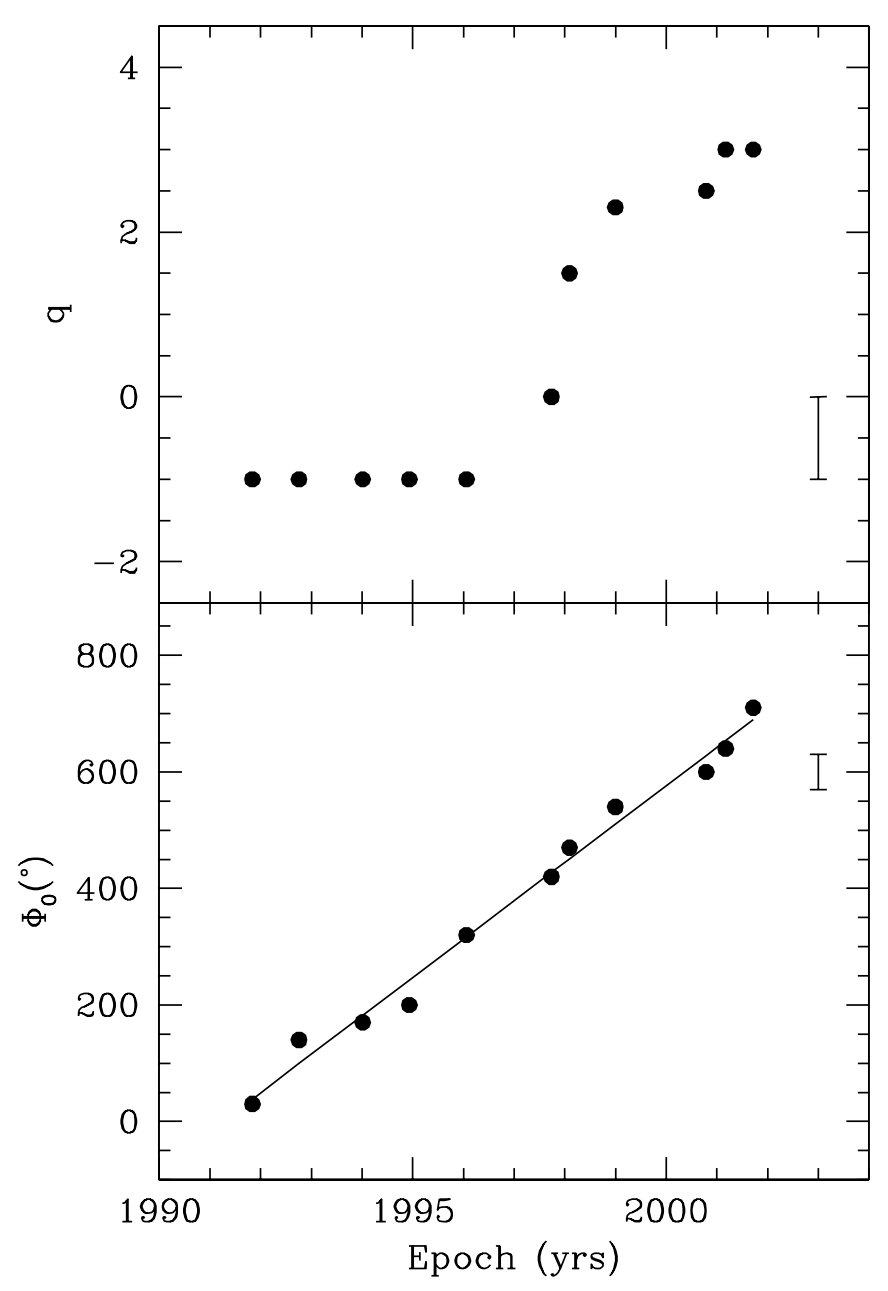

FIG. 13.-Evolution of the spiral-disk model parameters: emissivity-law power-law index $q$ (top) and orientation of the spiral arm relative to the line of sight $\phi_{0}$ (bottom) in the precessing scenario. The straight line shows a linear fit to the variation of $\phi_{0}$ with time.

The behavior of the $3 \mathrm{C} 390.3$ broad $\mathrm{H} \beta$ profile, as described by Shapovalova et al. (2001), would be similar to the case of NGC 1097 reported here (as discussed in $\S 4.1$ ), and they have proposed a similar interpretation: the flux

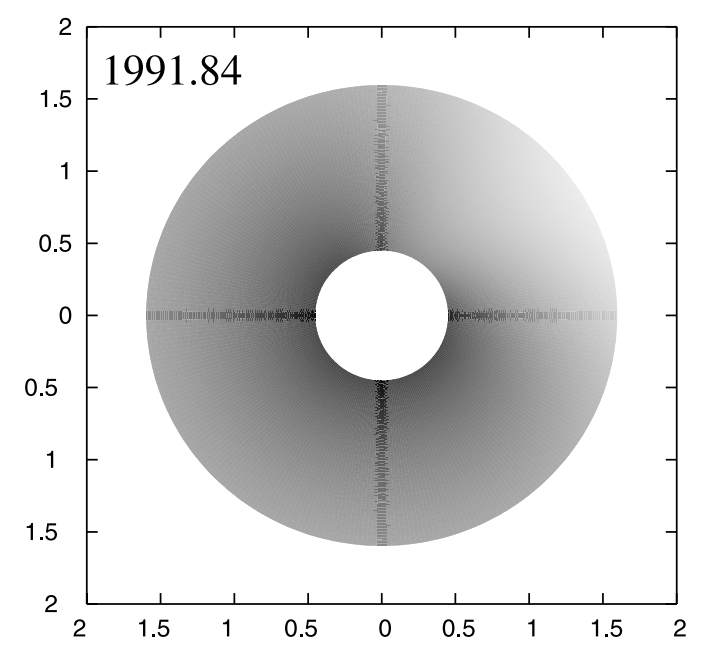

increase of $\mathrm{H} \beta$ is due to the increase in the source luminosity, which thus ionizes regions farther from the nucleus (lower velocity); the flux decrease of $\mathrm{H} \beta$ is due to a decrease in the ionizing luminosity, which shifts the region of maximum line emission closer to the center of the disk (higher velocity).

\subsection{Implications of the Observational Results and Models}

The pattern precession period inferred from the model fits can be connected to the mass of the central black hole. Although eccentric disks and spiral arms are related structures (see, for example, the illustration in Adams, Ruden, \& Shu 1989), in our application the precession rates are set by different mechanisms.

In the case of an eccentric disk, the precession rate is controlled by the relativistic advance of the pericenter. Therefore, we adapt the formula given by Weinberg (1972, p. 197), which we write as

$P_{\text {prec }}=\frac{2 \pi}{3} \frac{1+e}{(1-e)^{3 / 2}} \frac{G M}{c^{3}} \xi^{5 / 2}=10.4 \frac{1+e}{(1-e)^{3 / 2}} M_{6} \xi_{3}^{5 / 2} \mathrm{yr}$

where $M_{6}$ is the mass of the black hole in units of $10^{6} M_{\odot}$ and $\xi_{3}$ is the pericenter distance of the orbit in units of $10^{3} r_{g}$. If the outer, most eccentric ring of the disk is precessing as prescribed by the advance of pericenter, then $\xi_{3}=1.6$, $e=0.45$, and, for the period of $7.1 \mathrm{yr}$ obtained in $\S 4.2$, the resulting black hole mass is $6 \times 10^{4} M_{\odot}$. If the inner rings impose a faster precession rate, the resulting mass will be somewhat larger, with an upper limit corresponding to the innermost radius of $\xi_{3}=0.45$ and $e=0$, which yield a black hole mass of $5 \times 10^{6} M_{\odot}$.

In the case of the precessing spiral pattern, we can connect the precession period to the black hole mass using the results of Laughlin \& Korchagin (1996), who find that the pattern period is several times to an order of magnitude longer than the dynamical time. An extreme upper bound to the pattern period is the sound-crossing time of the disk. The dynamical and sound-crossing timescales are, respectively, given by

$$
\tau_{\text {dyn }}=2 M_{6} \xi_{3}^{3 / 2} \text { days }
$$

FIG. 14. - Same as Fig. 10, but for the circular disk-plus-spiral arm model 
and

$$
\tau_{s}=8 M_{6} \xi_{3} T_{5}^{-1 / 2} \text { months }
$$

(adapted from Frank et al. 1992), where now $\xi_{3}$ is the radius of the disk in units of $10^{3} r_{\mathrm{g}}$ and $T_{5}$ is the temperature in units of $10^{5} \mathrm{~K}$. By comparing these timescales with the precession period of $5.5 \mathrm{yr}$ inferred in $\S 4.3$, we estimate a black hole mass in the range $10^{7}-10^{8} \mathrm{M}$

Another scenario discussed briefly in SB97 involves the precession of a radiation-induced warp in the disk (Pringle 1996). In equation (4) of SB97, we estimated a precession period on the order of a decade, assuming a black hole mass of $10^{6} M_{\odot}$ and a mass of the accretion disk of $10^{-4} M_{\odot}$. Since this disk mass is, in fact, a lower limit (see SB97), the precession period determined above implies an upper limit on the black hole mass of $M<10^{6} M_{\odot}$ in the context of this picture.

All of the above scenarios can be assessed using a black hole mass derived from the velocity dispersion of the stars in the nucleus of NGC 1097. Emsellem et al. (2001) measure a velocity dispersion of $160 \pm 20 \mathrm{~km} \mathrm{~s}^{-1}$ in the inner $300 \mathrm{pc}$ of the bulge of NGC 1097. Using the mass-velocity dispersion relation of Tremaine et al. (2002), we infer a black hole mass in the range (2-9) $\times 10^{7} M_{\odot}$, where the uncertainty is dominated by the dispersion about the best-fitting relation (see Fig. 8 of Tremaine et al.). This black hole mass disfavors both the elliptical-disk and precessing-warp scenarios, leaving the spiral-arm scenario as the best candidate. The spiral-arm scenario is also favored by the fact that it provides the best description of the variable line profiles, by better "accommodating" the precession scenario, as described in $\S 4$.

\section{SUMMARY, CONCLUSIONS, AND OPEN QUESTIONS}

We have used spectroscopic observations of the broad, double-peaked $\mathrm{H} \alpha$ profile of the nucleus of NGC 1097 spanning the period between 1991 November and 2002 October in order to constrain and refine models for the variability of the line profile. The profiles display the following types of variations over these 11 years:

1. A smooth, long-term variation in the relative strengths of the blue and red peaks;

2. A decrease in the wavelength of the blue peak and a corresponding increase in the wavelength of the red peakin other words, a broadening of the double-peaked profile, suggesting that the bulk of the emission is shifting inward, to gas that is moving at higher velocities;

3. A decrease in the integrated luminosity of the line, so that in 2001 September it was approximately $\frac{1}{3}$ of its value in 1991 November;

4. An inverse correlation between the width and luminosity of the line;

5. A shift of the central wavelength of the profile (defined as the average wavelength of the two peaks), from approximately zero velocity relative to the narrow lines in 1991 to about $-450 \mathrm{~km} \mathrm{~s}^{-1}$ in 2001 .

The evolution of the double-peaked $\mathrm{H} \alpha$ profile of NGC 1097, described above, lends additional support to our earlier suggestion that the variations are a result of a precessing, nonaxisymmetric pattern in an accretion disk
(Eracleous et al. 1995; SB95; SB97). We have compared the observed line profiles with models of an eccentric (elliptical) disk and a disk with a spiral arm and have shown that both models can reproduce the data. However, we favor the spiral-arm model because (1) it can reproduce the evolution of the double-peaked profile as due to the precession of the spiral arm, while the elliptical-disk model cannot reproduce a smooth precession; and (2) it implies a black hole mass that is consistent with what is inferred from the radial velocity dispersion of the stars in the nucleus, while the ellipticaldisk model requires a mass that is an order of magnitude lower.

We have further found that the dimming of the broad, double-peaked $\mathrm{H} \alpha$ line is coupled with the increasing separation of the blue and red peaks. We have interpreted this behavior as a result of the dimming of the source of ionizing radiation, which causes the maximum emission region of the disk drift inward and thus to regions of higher orbital velocities. This dimming of the central source is progressing independently of dynamical phenomena in the disk, which is a reasonable outcome of the tidal disruption scenario that explains the abrupt appearance of the double-peaked lines. The coupling between the luminosity and width of the line seems to hold also at shorter timescales: an increase in the flux of the line is associated with a decrease in the line width, while a decrease in flux is associated with an increase in line width.

In the process of studying the evolution of the $\mathrm{H} \alpha$ profiles and fitting disk models to them, we found a gradual blueshift, especially at later epochs. This blueshift manifests itself as a gradual shift of the average wavelength of the two peaks. We have not attempted to model this effect, since our data so far cannot yield strong constraints. Nevertheless, we speculate that this blueshift may be associated with the onset of an accretion disk wind (Elvis 2000). Outflows with typical velocities of $1000 \mathrm{~km} \mathrm{~s}^{-1}$ _consistent with the blueshift of $\sim 400-500 \mathrm{~km} \mathrm{~s}^{-1}$ we have measured for NGC 1097 if we adopt an inclination of $34^{\circ}$ for the disk - have been frequently observed in the spectra of Seyfert galaxies as absorption lines in the UV (see, e.g., Kraemer, Crenshaw, \& Gabel 2001) and X-rays (the "warm absorber"), as well as in the emission lines of the narrow-line region. Models for these outflows attribute their origin in the accretion disk, to, for example, a hydromagnetically driven wind of discrete clouds (Emmering, Blandford, \& Shlosman 1992) or radiatively driven continuous winds (Murray et al. 1995; Proga, Stone, \& Kallman 2000). The presence of an outflow in the case of NGC 1097 is also consistent with observations of Fe II absorption lines in a recent HST UV spectrum (Eracleous 2002). Future observations of the variability of the Balmer line profiles should shed more light on the issue of the possible wind.

We thank Steinn Sigurdsson and Mario Livio for useful discussions on issues related to accretion disk dynamics. We acknowledge the Brazilian institutions CNPq, CAPES, and FAPERGS for partial support. This research was also supported in part by the NSF through grant AST 95-27289 and by NASA through grant NAG8-1755 to the University of Maryland. A. V. F.'s work is supported by NASA through grant GO-8684 from the Space Telescope Science Institute, which is operated by the Association of Universities for Research in Astronomy, Inc., under NASA contract 
NAS5-26555. We thank the staffs of the observatories used to obtain the spectra in this paper, as well as many associates who helped with the observations and reductions. The W. M. Keck Observatory is operated as a scientific partnership among the California Institute of Technology, the University of California, and NASA; the observatory was made possible by the generous financial support of the W. M. Keck Foundation.
Adams, F. C., Ruden, S. P., \& Shu, F. H. 1989, ApJ, 347, 959

Baptista, R., \& Catalán, M. S. 2000, ApJ, 539, L55

Barth, A. J., Ho, L. C., Filippenko, A. V., Rix, H.-W., \& Sargent, W. L. W. 2001, ApJ, 546, 205

Bower, G. A., Wilson, A. S., Heckman, T. M., \& Richstone, D. O. 1996, AJ, 111, 1901

Chakrabarti, S. K., \& Wiita, P. J. 1993, A\&A, 271, 216 . 1994, ApJ, 434, 518

Dumont, A. M., \& Collin-Souffrin, S. 1990, A\&A, 229, 313

Elvis, M. 2000, ApJ, 545, 63

Emmering, R. T., Blandford, R. D., \& Shlosman, I. 1992, ApJ, 385, 460

Emsellem, E., Greusard, D., Combes, F., Friedli, D., Leon, S., Pécontal, E., \& Wozniak, H. 2001, A\&A, 368, 52

Eracleous, M. 2002, in ASP Conf. Ser. 255, Mass Outflow in Active Galactic Nuclei: New Perspectives, ed. M. Crenshaw, S. B. Kraemer, \& I. M. George (San Francisco: ASP), 131

Eracleous, M., \& Halpern, J. P. 1998, ApJ, 505, 577

Eracleous, M., Halpern, J. P., Gilbert, A. M., Newman, J. A., \& Filippenko, A. V. 1997, ApJ, 490, 216

Eracleous, M., Livio, M., Halpern, J. P., \& Storchi-Bergmann, T. 1995, ApJ, 438, 610

Ferrarese, L., \& Merritt, D. 2000, ApJ, 539, L9

Frank, J., King, A. R., \& Raine, D. J. 1992, in Accretion Power in Astrophysics (2d ed.; Cambridge: Cambridge Univ. Press)

Gaskell, C. M. 1996, ApJ, 464, L107

Gebhardt, K., et al. 2000, ApJ, 539, L13

Gilbert, A. M., Eracleous, M., Filippenko, A. V., \& Halpern, J. P. 1999,

in ASP Conf. Ser. 175, Structure and Kinematics of Quasar Broad-

Line Regions, ed. C. M. Gaskell, W. N. Brandt, M. Dietrich,

D. Dultzin-Hacyan, \& M. Eracleous (San Francisco: ASP), 189

\section{RFERENCES}

Heckman, T. M. 1980, A\&A, 87, 152

Ho, L. C., Rudnick, G., Rix, H.-W., Shields, J. C., McIntosh, D. H., Filippenko, A. V., Sargent, W. L. W., \& Eracleous, M. 2000, ApJ, 541, 120

Kraemer, S. B., Crenshaw, D. M., \& Gabel, J. R. 2001, ApJ, 557, 30

Laughlin, G., \& Korchagin, V. 1996, ApJ, 460, 855

Murray, N., Chiang, J., Grossman, S. A., \& Voit, G. M. 1995, ApJ, 451, 498

Newman, J. A., Eracleous, M., Filippenko, A. V., \& Halpern, J. P. 1997, ApJ, 485, 570

Patterson, J., Halpern, J., \& Shambrook, A. 1993, ApJ, 419, 803

Pringle, J. E. 1996, MNRAS, 281, 357

Proga, D., Stone, J. M., \& Kallman, T. R. 2000, ApJ, 543, 686

Rees, M. J. 1988, Nature, 333, 523

Shapovalova, A. I., et al. 2001, A\&A, 376, 775

Shields, J. C., Rix, H.-W., McIntosh, D. H., Ho, L. C., Rudnick, G., Filippenko, A. V., Sargent, W. L. W., \& Sarzi, M. 2000, ApJ, 534, L27

Steeghs, D., Harlaftis, E., \& Horne, K. 1997, MNRAS, 290, L28

Storchi-Bergmann, T., Baldwin, J. A., \& Wilson, A. S. 1993, ApJ, 410, L11 (SB93)

Storchi-Bergmann, T., Eracleous, M., Livio, M., Wilson, A. S., Filippenko, A. V., \& Halpern, J. P. 1995, ApJ, 443, 617 (SB95)

Storchi-Bergmann, T., Eracleous, M., Ruiz, M. T., Livio, M., Wilson, A. S., \& Filippenko, A. V. 1997, ApJ, 489, 87 (SB97)

Tremaine, S., et al. 2002, ApJ, 574, 740

Weinberg, S. 1972, Gravitation and Cosmology: Principles and Applications of the General Theory of Relativity (New York: Wiley)

Zheng, W., Veilleux, S., \& Grandi, S. A. 1991, ApJ, 381, 418 